\title{
Parasitoides do bicudo Anthonomus grandis e predadores residentes em algodoeiro pulverizado com caulim
}

\author{
Parasitoids of boll weevil Anthonomus grandis and resident \\ predators in kaolin-treated cotton
}

\author{
Roberta Leme dos Santos ${ }^{1}$; Robério Carlos dos Santos Neves²; \\ Felipe Colares ${ }^{2}$; Jorge Braz Torres ${ }^{3 *}$
}

\begin{abstract}
Resumo
O uso simultâneo de diferentes métodos de controle é fundamental para o sucesso no manejo de pragas. Esse estudo investigou o efeito de aplicações do caulim nas populações de predadores residentes no dossel do algodoeiro e no parasitismo do bicudo em estruturas coletadas do solo em campo e, em botões florais em laboratório. A abundância de predadores residentes no dossel das plantas incluindo Araneae, Formicidae, Chrysopidae e Coccinellidae foi similar entre as áreas tratadas e não tratadas com o caulim, bem como a emergência dos parasitoides Bracon vulgaris Ashmead (Hymenoptera: Braconidae) e Catolaccus grandis Burks (Hymenoptera: Pteromalidae) oriundos de estruturas coletadas no solo em ambas as áreas. Da mesma forma, o parasitismo do bicudo em botões tratados e não tratados com caulim foi semelhante quando foram oferecidos ao parasitoide $B$. vulgaris em testes com chance de escolha. Sendo assim, os resultados mostram que aplicações do caulim não afetam o controle biológico natural por parasitoides associados ao bicudo e lagarta rosada e predadores residentes no algodoeiro.

Palavras-chave: Controle físico, controle biológico, Anthonomus grandis e Pectinophora gossypiella
\end{abstract}

\begin{abstract}
Simultaneous use of control methods is essential to reach success in managing arthropod pests. The current study investigated the effect of kaolin application on resident predators in the cotton plant canopy and parasitism of boll weevil on abscised squares in the field, and parasitism of boll weevil in the laboratory. Predators Araneae, Formicidae, Chrysopidae, and Coccinellidae showed similar seasonal densities for kaolin-treated and untreated cotton fields as well as the emergence rate of the parasitoids Bracon vulgaris Ashmead (Hymenoptera: Braconidae) and Catolaccus grandis Burks (Hymenoptera: Pteromalidae) from abscised field-collected structures. Under laboratory conditions, the parasitism of boll weevil larvae infesting squares was similar when treated and untreated squares with kaolin were offered to the parasitoid under free choice test. Therefore, the results show that spraying cotton fields with kaolin does not affect the natural biological control by parasitoids of boll weevil and pink bollworm and resident predators naturally occurring in cotton fields.
\end{abstract}

Key words: Physical control, biological control, Anthonomus grandis e Pectinophora gossypiella

\footnotetext{
${ }^{1}$ Discente de Doutorado, Dept ${ }^{0}$ de Fitotecnia, Universidade Federal de Viçosa, UFV, Campus Universitário, Viçosa, MG. E-mail: robertaleme2@uol.com.br

${ }^{2}$ Discentes de Doutorado, Programa de Pós-Graduação em Entomologia Agrícola, Universidade Federal Rural de Pernambuco, UFRRJ, Recife, PE. E-mail: roberiocneves@yahoo.com.br; felipecolares@hotmail.com

${ }^{3}$ Prof. da Universidade Federal Rural de Pernambuco, Programa de Pós-Graduação em Entomologia Agrícola, PPGEA/UFRPE, Recife, PE. E-mail: jtorres@depa.ufrpe.br

* Autor para correspondência
} 


\section{Introdução}

A incidência do bicudo-do-algodoeiro, Anthonomus grandis Boh. (Coleoptera: Curculionidae) é um fator limitante para o cultivo do algodoeiro, especialmente, em cultivo orgânico (TORRES; RUBERSON; WHITEHOUSE, 2009). Se comparado às demais pragas do algodoeiro, o bicudo possui baixa mortalidade por inimigos naturais (RAMALHO; WANDERLEY, 1996).

No Brasil, são citadas 13 espécies de parasitoides e 10 espécies de predadores atuando como agentes de controle biológico natural do bicudo na cultura do algodoeiro (RAMALHO; WANDERLEY, 1996). Os principais parasitoides do bicudo são Bracon vulgaris Ashmead (Hymenoptera: Braconidae) e Catolaccus grandis Burks (Hymenoptera: Pteromalidae) (MORALES-RAMOS et al., 1998; RAMALHO et al., 2000), sendo que o primeiro também parasita e se desenvolve em larvas da lagarta rosada. Se comparado às demais pragas da cultura, constata-se que existem poucos inimigos naturais associados ao bicudo (WHITCOMB; BELL, 1964; BASTOS; TORRES, 2006). No entanto, para qualquer método de controle compondo o manejo integrado de pragas preconiza-se que esse seja compatível com os agentes de controle biológico.

Tornar a planta de algodoeiro menos favorável a colonização do bicudo é uma tática vantajosa devido à dificuldade de controle do mesmo após o seu estabelecimento, mesmo utilizando inseticidas, os quais são na maioria de largo espectro. Assim, a pulverização da lavoura com o caulim vem sendo testada visando à redução das infestações da lavoura pelo bicudo (SHOWLER, 2002; SILVA; RAMALHO, 2013). O caulim é um produto deterrente que pode repelir diretamente ou tornar as plantas hospedeiras irreconhecíveis ou impróprias para sítios de alimentação e oviposição (GARCIA; BERKETT; BRADSHAW, 2003). A atratividade do bicudo pela folhagem das plantas cobertas por caulim é reduzida em relação às plantas sem essa cobertura, em chance de escolha (SHOWLER, 2002). Esse efeito pode ser explorado como alternativa para reduzir a colonização da lavoura, inclusive em associação com outras técnicas de manejo, como a possibilidade do uso do caulim pulverizado no interior da lavoura associado à pulverização de inseticida convencional nas bordaduras (SILVA; RAMALHO, 2013).

O filme formado pelo caulim, além de ser barreira física que dificulta a aceitação da planta por insetos herbívoros, deixa a planta com coloração branca brilhante podendo torná-la repelente ou mesmo irreconhecível pelo inseto (SHOWLER, 2002). Além de dificultar o reconhecimento da planta, seja na forma visual ou tátil, o caulim pode ocasionar mortalidade da praga por sufocamento com a obstrução dos espiráculos e por dessecação (GLENN; PUTERKA, 2005).

Tornar a planta de algodoeiro menos favorável a colonização do bicudo é uma tática vantajosa devido à dificuldade de controle do mesmo após o seu estabelecimento na lavoura. Assim, a pulverização da lavoura com o caulim vem sendo testada visando à redução das infestações do bicudo (SHOWLER, 2002; SILVA; RAMALHO, 2013). A atratividade do bicudo pela folhagem das plantas cobertas por caulim é reduzida em relação às plantas sem essa cobertura (SHOWLER, 2002). Esse efeito pode ser explorado como alternativa para reduzir a colonização da lavoura, inclusive em associação com outras técnicas de manejo (SILVA; RAMALHO, 2013). No entanto, para qualquer controle compondo o manejo integrado de pragas preconiza-se que esse seja compatível com os agentes de controle biológico. Populações de predadores e parasitoides em pomares de oliva foram reduzidas após pulverizações com caulim em comparação às áreas sem aplicação (GONZÁLEZNÚÑEZ et al., 2008). Em algodoeiro, populações do percevejo Orius, vespas e dípteros predadores foram reduzidas em uma avaliação do tratamento com caulim (SHOWLER; SÉTAMOU, 2004). Assim, este trabalho investigou o efeito de aplicações do caulim no parasitismo do bicudo em laboratório, 
nas estruturas coletadas em campo e nas populações de inimigos naturais residentes no dossel da planta durante duas safras.

\section{Material e Métodos}

Os experimentos de campo foram conduzidos durante as safras 2010 e 2011, em propriedade particular cedida para experimentação, localizada em Surubim, PE (0753'48,9' S e 3549'19,2” W), enquanto o experimento de laboratório foi conduzido no laboratório de Controle Biológico de Insetos da Universidade Federal Rural de Pernambuco (UFRPE). Dois tratamentos foram conduzidos: plantas pulverizadas com caulim diluído em água na concentração de $60 \mathrm{~g} / \mathrm{L}$ (+caulim) e plantas não pulverizadas (-caulim), em delineamento de blocos ao acaso com quatro repetições. Cada repetição foi composta por 10 linhas de 12 metros de comprimento de algodoeiro da variedade BRS Rubi de fibra marrom, separadas por quatro fileiras de milho Híbrido AG 122.

Os plantios foram realizados em 10/04/2010 e em 30/05/2011. Foi adotado o espaçamento de um metro entre linhas, tradicional na região devido ao controle de plantas invasoras com cultivador de tração animal, e densidade de cinco a seis plantas por metro linear. A precipitação, temperatura e a umidade relativa foram medidas empregando pluviômetro e DataLogger Hobo ${ }^{\circledR}$ (Onset Computer Coorp.) instalados no local. No período do estudo, de abril a agosto de 2010 e de maio a setembro de 2011 foram registradas (médias do período \pm DP) temperaturas e umidade relativa do ar de 23,1 \pm $4,7^{\circ} \mathrm{C}$ e $22,8 \pm 4,5^{\circ} \mathrm{C}, 49 \pm 25,9 \%$ e $74,5 \pm 8,4 \%$, e precipitação acumulada $602 \mathrm{~mm}$ e $1239 \mathrm{~mm}$, respectivamente.

As aplicações do caulim (Caulisa, Campina Grande, PB) foram feitas em intervalos de oito dias, sendo iniciadas aos 47 dias após o plantio (DAP) nas duas safras. O caulim foi pulverizado a $60 \mathrm{~g} / \mathrm{L}$ mais espalhante adesivo Will Fix ${ }^{\circledR}$ a $0,025 \%$, utilizando pulverizador costal manual Jacto ${ }^{\circledR} \mathrm{PJH}$
20L, com bico do tipo cone vazio. O volume de calda variou de 417 a $834 \mathrm{~L} / \mathrm{ha}$, de acordo com o desenvolvimento das plantas.

\section{Parasitismo em estruturas reprodutivas} caídas ao solo. Estruturas reprodutivas caídas ao solo foram coletadas quando se observou o início da queda aos 47 DAP em 2010 e aos 62 DAP em 2011, respectivamente, em ambos os tratamentos + caulim e -caulim. Foram realizadas oito coletas em 2010 e 10 coletas em 2011 em intervalos de cinco a 10 dias. As estruturas coletadas foram levadas ao laboratório e separadas em botões florais e maçãs.

Amostras aleatórias de 200 botões florais e 60 maçãs a partir do material coletado em campo foram acondicionadas em gaiolas confeccionadas de garrafas tipo PET, com aberturas laterais (seis milímetros) fechadas com tela (um milímetro) e identificadas quanto aos tratamentos, repetições e datas de coleta. A abertura superior das gaiolas foi fechada com tecido organza preso com fita adesiva, permitindo a evaporação de parte da umidade presente nas estruturas, especialmente maçãs.

A emergência de parasitoides e das pragas associadas (bicudo e lagarta rosada) foi avaliada aos 5,10 e 15 dias após o confinamento do material, para evitar qualquer perda de inseto por apodrecimento ou escape. Entretanto, como resultado foi considerado o total de insetos por repetição das três avaliações. O material foi mantido em condições de laboratório a $25 \pm 2{ }^{\circ} \mathrm{C}$, fotoperíodo de $12 \mathrm{~h}$ e umidade relativa de $65 \pm 5 \%$. A taxa de emergência de parasitoides por repetição foi obtida pela correção em função do total de estruturas coletadas (botões florais e maçãs) e estruturas monitoradas para a emergência. Para a comparação entre os tratamentos +caulim e -caulim, foi estimada a taxa de parasitismo aparente (TPA): TPA $=[$ (no. de parasitoides emergidos/ (no. de parasitoides + no. de hospedeiros-praga emergidos)]. A TPA foi empregada, pois não se sabia a quantidade de hospedeiros disponíveis para o parasitismo (desenvolvimento endofítico), tendo-se em vista que nem todos os botões florais 
atacados teriam hospedeiros viáveis. Além disso, nem todos os hospedeiros parasitados produzem obrigatoriamente um parasitoide adulto e existe a possibilidade de mortalidade natural ocasionada por outro fator que não fosse o parasitismo. Para a taxa de parasitismo por B. vulgaris foi considerado o bicudo mais a lagarta rosada como hospedeiros, e apenas o bicudo para o parasitismo por C. grandis, devido à especificidade deste último parasitoide (BURKS, 1954). Em seguida, a TPA foi submetida aos testes de Lavene e Bartlett para a normalidade e homogeneidade de variância, respectivamente, e quando necessário, procedeu-se com a transformação de dados. Para a comparação dos tratamentos entre si e no tempo (datas de coleta) foi realizada análise de variância, considerando-se o procedimento PROC ANOVA do SAS (SAS INSTITUTE, 2001) com medidas repetidas no tempo (coletas sucessivas realizadas na mesma área/repetição).

Abundância de predadores em plantas com caulim. A incidência de predadores foi monitorada em avaliações que iniciaram aos 49 DAP em 2010 e aos 39 DAPem 2011, respectivamente. As avaliações de predadores no dossel da planta foram realizadas em intervalos de oito dias, no período da manhã, antes da pulverização com o caulim (tratamento +caulim) e simultaneamente no tratamento caulim. Em 2010 e 2011 foram realizadas nove e 11 avaliações, respectivamente, sendo encerradas quando ocorreu o início da abertura de capulhos. A amostragem foi visual e realizada em 10 plantas ao acaso por repetição, sendo consideradas três folhas expandidas do ponteiro como unidade amostral. Foi quantificada a incidência de joaninhas, bicholixeiro, aranhas e formigas, bem como de pulgões. Os resultados foram considerados como média de predadores em 10 plantas avaliadas por repetição. Os resultados foram submetidos à análise de variância, empregando-se o procedimento de medidas repetidas no tempo para a comparação dos tratamentos entre si e no tempo (diferentes datas de amostragem no mesmo tratamento). Também, a relação entre a abundância da presa pulgão e predadores, bem como entre os predadores foi testada mediante pares de correlações através do método de correlação de Pearson $(r)$ e interpretada a $5 \%$ de probabilidade, usando o PROC CORR do SAS (SAS INSTITUTE, 2001).

Parasitismo do bicudo por Bracon vulgaris em botões florais pulverizados com o caulim em laboratório. $\mathrm{O}$ parasitismo do bicudo por $B$. vulgaris em botões florais pulverizados ou não com o caulim foi estudado em laboratório empregandose fêmeas do parasitoide obtidas da criação mantida no Laboratório de Controle Biológico de Insetos da UFRPE. As fêmeas do parasitoide foram utilizadas com cinco dias de idade. Para os testes, foram empregados botões florais com larvas do bicudo em desenvolvimento no seu interior. Os botões foram selecionados e fixados através do pedúnculo em plataformas de isopor de $3 \mathrm{~cm}$ de largura e 36 $\mathrm{cm}$ de comprimento, em um total de 10 botões por plataforma. Após fixados nas plataformas, os botões foram pulverizados com caulim a $60 \mathrm{~g} / \mathrm{L}$ e espalhante adesivo, sendo realizadas duas pulverizações com pulverizador manual de um litro de capacidade em intervalo de duas horas para se obter total cobertura do botão floral e das brácteas. Em seguida, os botões pulverizados foram deixados para secar a calda durante uma noite e expostos aos parasitoides no início da fotofase do dia seguinte. As plataformas com botões florais representando o tratamento sem caulim foram pulverizadas apenas com o espalhante adesivo.

A exposição dos botões florais +caulim e-caulim às fêmeas do parasitoide foi realizada empregandose arenas compostas por gaiolas de acrílico de $35 \times 40 \times 50 \mathrm{~cm}$ (largura, altura, comprimento). As plataformas contendo botões +caulim e -caulim foram fixadas a $10 \mathrm{~cm}$ do teto e distando $30 \mathrm{~cm}$ uma da outra. Em cada gaiola foram liberadas cinco fêmeas do parasitoide, as quais tinham para escolha 10 botões florais pulverizados com caulim e 10 botões florais sem caulim durante $24 \mathrm{~h}$ ( $12 \mathrm{~h}$ de luz e $12 \mathrm{~h}$ de escuro). As gaiolas foram acomodadas sobre bancadas em laboratório com iluminação realizada 
por luz branca $\sim 650$ lux incidente do teto. Após a exposição ao parasitismo, os botões foram mantidos isolados em câmara do tipo BOD regulada a $25^{\circ} \mathrm{C}$, fotofase de $12 \mathrm{~h}$ e $60 \%$ de UR, e determinada a taxa de parasitismo com base na emergência de parasitoides.

O experimento foi conduzido em blocos ao acaso no tempo com chance de escolha para parasitismo entre botão floral pulverizado ou não com o caulim. Cada gaiola foi considerada como uma repetição e ao total foram realizadas 18 repetições, arranjadas em quatro blocos (data de instalação) contendo quatro, cinco, quatro e cinco repetições cada. Assim, o efeito do caulim na escolha e parasitismo por $B$. vulgaris foi testado mediante a hipótese de igualdade de parasitismo (50\%:50\%) entre os tratamentos pelo PROC FREQ do SAS e interpretados pelo teste do qui-quadrado ( $\mathrm{c}^{2}$ ) (SAS INSTITUTE, 2001).

\section{Resultados e Discussão}

Estruturas caídas ao solo na safra 2010 corresponderam a 1.775 e 1.986 maçãs recém- formadas (aproximadamente um centímetro de diâmetro) e 6.176 e 11.539 botões florais nas parcelas +caulim e -caulim, respectivamente. Estruturas caídas ao solo na safra 2011 corresponderam a 1.242 e 1.354 maçãs e 677 e 1.589 botões florais nas parcelas +caulim e -caulim, respectivamente. A emergência de parasitoides restringiu-se a duas espécies: B. vulgaris e C. grandis. Estes parasitoides emergiram tanto de maçãs recém-formadas como de botões florais coletados do solo e resultando em similar TPA em algodoeiro pulverizado ou não com o caulim em ambas as safras (Tabela 1). Parasitismo por B. vulgaris foi registrado a partir da segunda coleta em 10 de julho e o pico de parasitismo na coleta em 20 de julho de 2010 (101 DAP) (Figura 1). O parasitoide $C$. grandis teve a presença registrada a partir de 10 de julho com máxima ocorrência em 19 de agosto. Em 2011, o parasitismo por $B$. vulgaris foi registrado a partir da segunda coleta em 8 de agosto e o pico de parasitismo em 28 de agosto (90 DAP). O parasitoide $C$. grandis teve a presença registrada a partir de 5 de setembro com máxima ocorrência em 17 de setembro (Figura 1).

Tabela 1. Médias ( \pm EP) da taxa de parasitismo aparente (TPA) em botões florais coletados do solo em algodoeiro pulverizado ou não com o caulim durante as safras de 2010 e 2011, Surubim, PE.

\begin{tabular}{lcccc}
\hline & \multicolumn{2}{c}{ TPA Safra 2010 } & \multicolumn{2}{c}{ TPA Safra 2011 } \\
\cline { 2 - 5 } Tratamentos & Bracon vulgaris & Catolacus grandis & Bracon vulgaris & Catolacus grandis \\
\hline+ caulim & $2,3 \pm 0,90$ & $0,55 \pm 0,32$ & $10,09 \pm 5,47$ & $0,23 \pm 0,14$ \\
- caulim & $2,4 \pm 0,65$ & $0,24 \pm 0,15$ & $20,98 \pm 2,1$ & $1,39 \pm 0,78$ \\
\hline Teste de $t^{\text {valor de P }}$ & $2,15^{0,5453}$ & $4,50^{0,2481}$ & $-1,85^{0,1130}$ & $1,48^{0,2313}$ \\
\hline
\end{tabular}

${ }^{1}$ [(número de parasitoides emergidos/(número de parasitoides + número de hospedeiro-praga emergidos) x 100].

Fonte: Elaboração dos autores.

A abundância média de predadores em 10 plantas de algodoeiro avaliadas por parcela, como imaturos de Coccinellidae e Chrysopidae, imaturos e adultos de Araneae e adultos de Formicidae não diferiu nos tratamentos +caulim e -caulim $(\mathrm{P}>0,05)$, exceto para Chrysopidae que foi superior no tratamento +caulim na primeira avaliação em $2010\left(\mathrm{~F}_{8,24}=\right.$ 2,96; $\mathrm{P}=0,0186$ ) (Figura 2). A abundância de larvas de Chrysopidae, predominantemente, Chrysoperla externa Hagen foi de 5,5 $\pm 1,3$ e 1,0 0 0,7 indivíduos em 10 plantas nos tratamentos +caulim e -caulim, respectivamente. Para Formicidae, foi observada tendência à maior abundância nas duas primeiras avaliações no tratamento -caulim, porém somente foi significativa em favor ao tratamento - caulim na avaliação 20 de agosto quando não foi encontrado formigas no tratamento +caulim. 
Figura 1. Taxa de parasitismo aparente (+EP) para os parasitoides Bracon vulgaris e Catolaccus grandis em botões florais coletados do solo em algodoeiro com e sem pulverização com o caulim nas safras de 2010 e 2011. Surubim, PE.
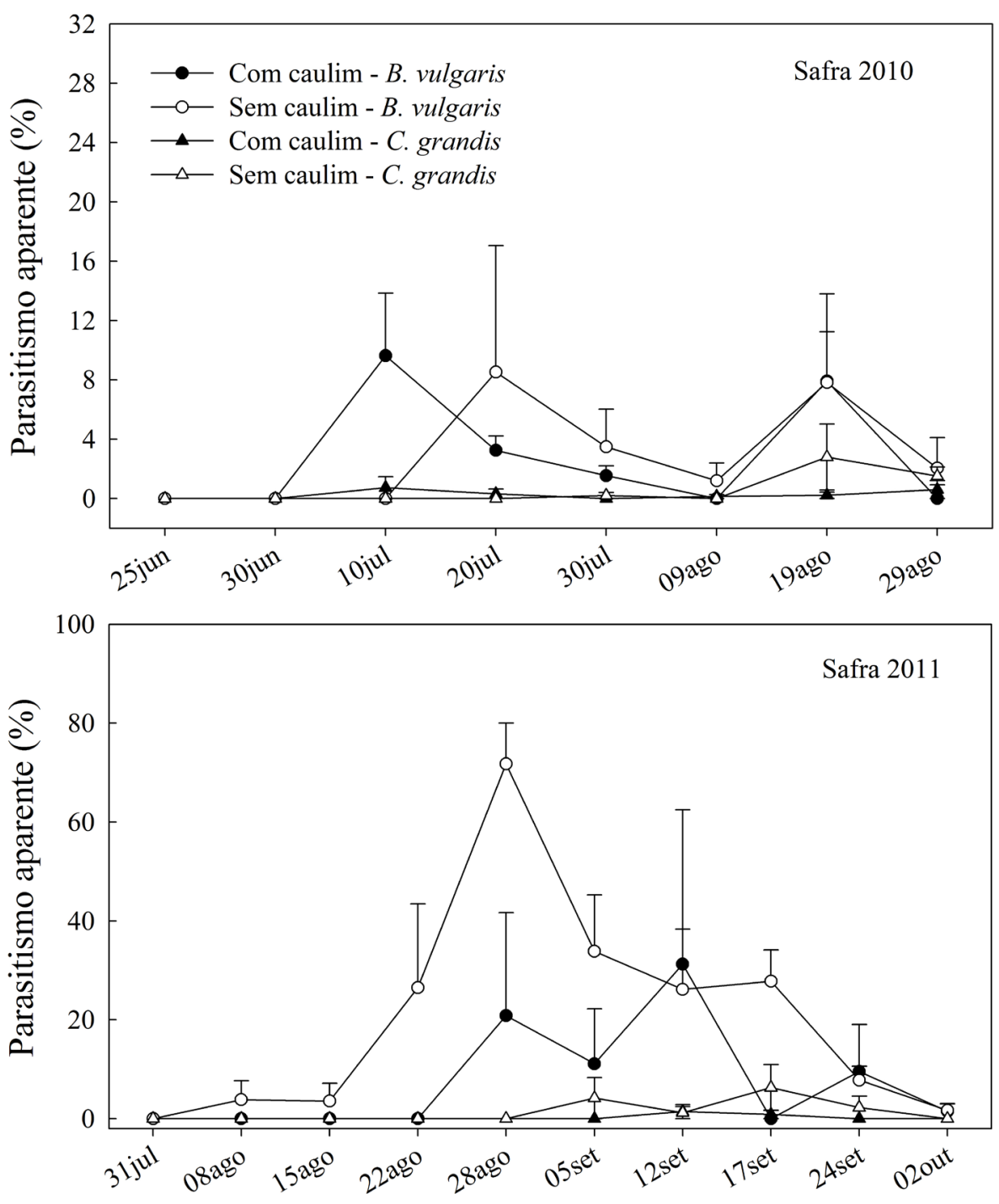

Fonte: Elaboração dos autores.

Coccinellidae foi representada pelas espécies de frequente ocorrência em algodoeiro como Zagreus bimaculosus Mulsant, Cycloneda sanguinea L. e Eriopis connexa Germar, além de espécies de Scymnus spp. (BASTOS; TORRES, 2006). A abundância média de larvas de Coccinellidae foi similar entre os tratamentos com e sem caulim (Figura 2). O mesmo foi observado para Araneae com predominância de Cheiracanthium sp. No entanto, a ocorrência dos predadores foi variável em função das avaliações para Chrysopidae $\left(\mathrm{F}_{8,24}=\right.$ $6,69 ; \mathrm{P}<0,0001)$, Araneae $\left(\mathrm{F}_{8,24}=7,67 ; \mathrm{P}<0,0001\right)$ e, apenas, parcialmente para Formicidae $\left(\mathrm{F}_{8,24}=\right.$ $2,29 ; \mathrm{P}=0,0555)$ e Coccinellidae $\left(\mathrm{F}_{8,24}=2,00 ; \mathrm{P}=\right.$ 0,0899) (Figura 1). As correlações da abundância de predadores e do pulgão Aphis gossypii Glover (Hemiptera: Aphididae), revelaram-se positivas e significativas para Coccinellidae em ambos 
os tratamentos, e para Araneae e Fomicidae no tratamento -caulim (Tabela 2). A abundância de Formicidae apresentou relação positiva com a abundância de Araneae e Coccinellidae nos tratamentos +caulim e -caulim, respectivamente. As demais correlações foram positivas, exceto a relação entre Chrysopidae e pulgões no tratamento -caulim que foi negativa, porém não significativa.

Figura 2. Número médio (+ EP) de Araneae, Coccinellidae, Chrysopidae e Formicidae, em algodoeiro pulverizado ou não com o caulim nas safras de 2010 e 2011, Surubim, PE. Indica diferença significativa entre os tratamentos com e sem caulim nas respectivas datas a $5 \%$ de probabilidade.

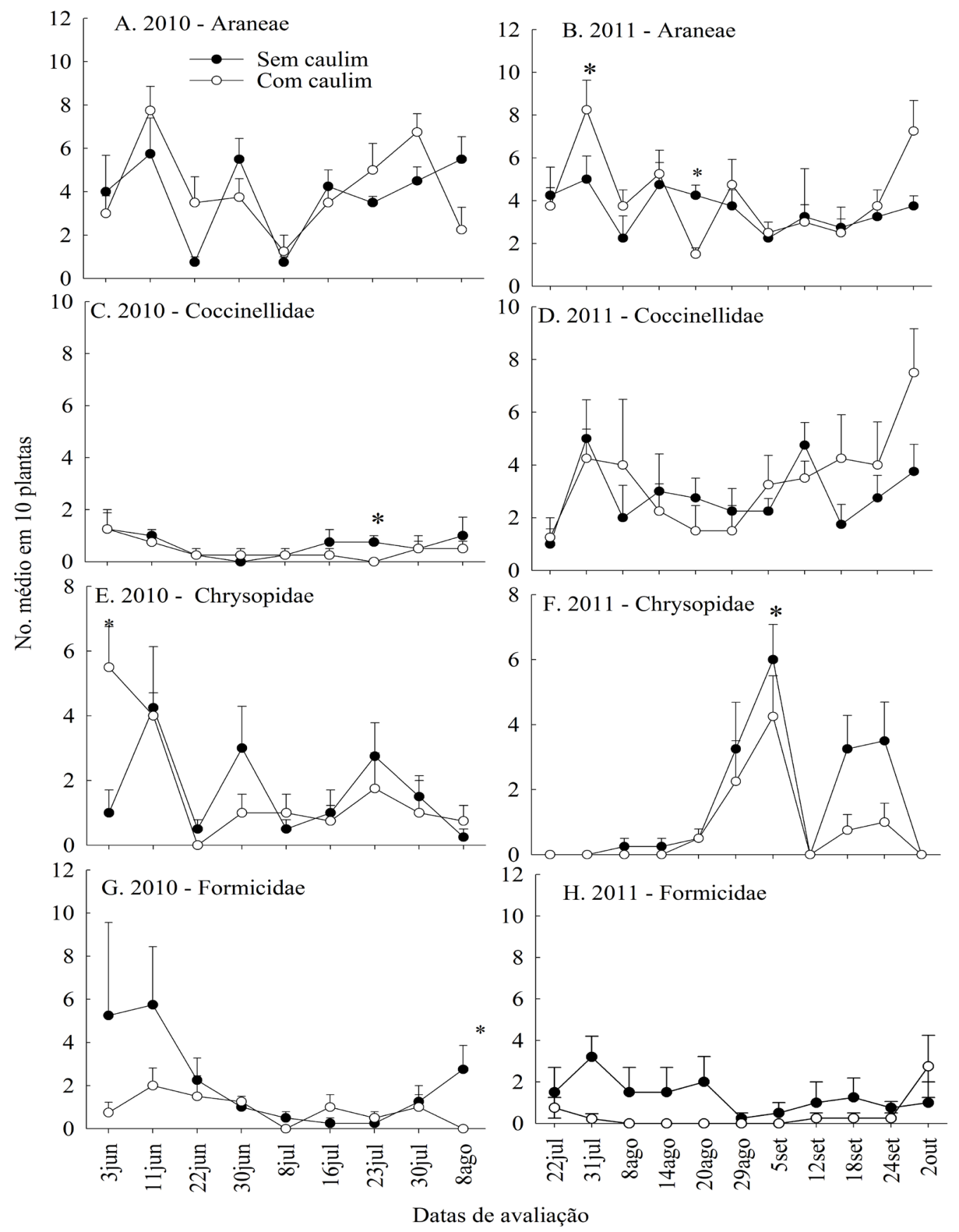

Fonte: Elaboração dos autores. 
Tabela 2. Coeficientes de correlação para os pares de comparação da abundância de pulgão e predadores residentes (larvas de Coccinellidae e Chrysopidae, adultos de Araneae e Formicidae) em algodoeiro com e sem pulverização com o caulim, nas safras de 2010 e 2011. Surubim, PE.

\begin{tabular}{|c|c|c|c|c|}
\hline & Coccinellidae & Chrysopidae & Araneae & Formicidae \\
\hline Safra 2010 & \multicolumn{4}{|c|}{ Com Caulim } \\
\hline Aphididae $^{1}$ & $0,4377^{0,0076^{*}}$ & $0,1542^{0,3691}$ & $0,1476^{0,3902}$ & $-0,0856^{0,6193}$ \\
\hline Coccinellidae & & $0,2189^{0,1995}$ & $0,0519^{0,7636}$ & $0,1873^{0,2740}$ \\
\hline Chrysopidae & & & $0,1731^{0,3126}$ & $0,1254^{0,4660}$ \\
\hline \multirow[t]{2}{*}{ Araneae } & & & & $0,3196^{0,0574}$ \\
\hline & \multicolumn{4}{|c|}{ Sem Caulim } \\
\hline Aphididae & $0,5046^{0,0017^{*}}$ & $-0,0069^{0,9678}$ & $0,3675^{0,0274}$ & $0,5626^{0,0004^{*}}$ \\
\hline Coccinellidae & & $0,0559^{0,7457}$ & $0,0546^{0,7518}$ & $0,4782^{0,0032^{*}}$ \\
\hline Chrysopidae & & & $0,0905^{0,5994}$ & $0,0138^{0,9363}$ \\
\hline \multirow[t]{2}{*}{ Araneae } & & & & $0,2407^{0,1577}$ \\
\hline & Coccinellidae & Chrysopidae & Araneae & Formicidae \\
\hline Safra 2011 & \multicolumn{4}{|c|}{ Com Caulim } \\
\hline Aphididae $^{1}$ & $0,2792^{0,0547}$ & $0,1167^{0,4296}$ & $-0,0315^{0,8318}$ & $0,2789^{0,0549}$ \\
\hline Coccinellidae & & $0,0076^{0,9590}$ & $0,2919^{0,0441^{*}}$ & $0,6899<<0,0001^{*}$ \\
\hline Chrysopidae & & & $-0,1117^{0,4499}$ & $-0,1173^{0,4271}$ \\
\hline \multirow[t]{2}{*}{ Araneae } & & & & $0,2005^{0,1719}$ \\
\hline & \multicolumn{4}{|c|}{ Sem Caulim } \\
\hline Aphididae & $-0,0314^{0,8321}$ & $0,2041^{0,1641}$ & $-0,1168^{0,4293}$ & $-0,0674^{0,6492}$ \\
\hline Coccinellidae & & $-0,1111^{0,4524}$ & $0,3333^{0,0203^{*}}$ & $0,4487^{0,0014^{*}}$ \\
\hline Chrysopidae & & & $-0,1525^{0,3007}$ & $-0,06701^{0,6507}$ \\
\hline Araneae & & & & $0,1825^{0,2144}$ \\
\hline
\end{tabular}

${ }^{1}$ Coeficientes de correlação de Pearson $(r)$ e valor de $P$-estatístico sobrescrito. *Indica significância a $5 \%$ de probabilidade.

Fonte: Elaboração dos autores.

A ocorrência de predadores residentes na safra de 2011 também não demonstrou variabilidade na abundância entre os tratamentos +caulim e -caulim $(\mathrm{P}>0,05)$, exceto para Araneae. Este grupo de predadores apresentou diferença entre os tratamentos na segunda e quinta avaliações, tendo interação significativa tratamento versus datas de avaliação (ANOVA; $\mathrm{F}_{10,30}=4,01 ; \mathrm{P}=0,0015$ ) (Figura 2), sendo que na segunda avaliação a ocorrência para o tratamento + caulim foi superior $(8,25 \pm 1,38)$ comparado ao -caulim $(5,00 \pm 1,08),\left(\right.$ ANOVA; $F_{1,10}$ $=46,09 ; \mathrm{P}=0,0065) \mathrm{e}$, na quinta avaliação superior para o tratamento -caulim $(4,25 \pm 0,48)$ comparado ao +caulim (1,50 $\pm 0,29),\left(\right.$ ANOVA; $\mathrm{F}_{1,10}=33,00 ; \mathrm{P}$ $=0,105)$. A abundância de Chrysopidae apresentou diferença entre tratamentos na sétima avaliação, tendo interação significativa tratamento versus datas de avaliação (ANOVA; $\mathrm{F}_{10,30}=2,72 ; \mathrm{P}=0,0166$ ), sendo superior no tratamento -caulim comparado ao tratamento +caulim $(6,0 \pm 1,08$ versus $4,25 \pm$ 1,25 indivíduos). Por outro lado, Coccinellidae e Formicidae apresentaram abundância média similar entre os tratamentos em todas as avaliações na safra 2011 (Figura 2).

As correlações com Coccinellidae, Araneae e Fomicidae revelaram-se positivas e significativas para ambos os tratamentos em 2011 (Tabela 2). Chrysopidae e Formicidae, Chrysopidae e Araneae apresentaram relações negativas, porém não significativas entre os tratamentos, mesmo resultado verificado entre Chrysopidae e Coccinellidae. Quanto à abundância de predadores em relação à da presa $A$. gossypii, foram observadas correlações negativas nas comparações entre presa e Araneae para ambos os tratamentos, e no tratamento -caulim entre presa e Coccinellidae, e 
entre presa e Formicidae, mas não significativas. Essas correlações positivas podem ser resultantes da interação direta entre predadores e pragas como presas sendo importantes para o manejo integrado de pragas. Mas, também, podem ocorrer entre predadores intraguilda e presas intraguilda como é comum em agroecossistemas onde a fomiga lavapé e aranhas comportam-se como predadores intraguilda de ovos, larvas e pupas de outros predadores como Chrysopidae e Coccinellidae, sendo estes últimos utilizados como presas intraguilda (Tabela 2).

A escolha de B. vulgaris para parasitar o bicudodo-algodoeiro não diferiu entre botões florais pulverizados ou não com o caulim. $\mathrm{O}$ parasitismo obtido em cada bloco de resultados, bem como para a média geral de parasitismo não foi diferente $\left(\chi^{2}=0,081 ; \mathrm{P}=0,7751 ; \mathrm{gl}=1\right)$. No geral, foram obtidos em média ( \pm IC a 95\%) 12,7 $\pm 3,75$ e 11,75 \pm $8,15 \%$ de parasitismo em botões florais pulverizados com caulim e sem caulim, respectivamente.

A taxa média de parasitismo em campo não foi afetada pela aplicação do caulim nas duas safras, bem como em estudo de escolha em laboratório. Sendo assim, o caulim é um produto que não interfere na ocorrência dos parasitoides comuns na região e que oferecem controle natural do bicudo. Liberações iniciais do parasitoide $C$. grandis no Agreste, Sertão e regiões do Seridó, PB, juntamente com B. vulgaris ocasionaram mortalidade natural da praga entorno de 10\% (RAMALHO; SILVA, 1993). Liberações com o equivalente a 700 adultos de C. grandis por hectare resultou em até $83 \%$ de redução na infestação do bicudo segundo Ramalho et al. (2000). Adams, Cross e Mitchell (1969) realizaram a liberação equivalente a 1977 fêmeas de Bracon mellitor Say (Hymenoptera: Braconidae) por hectare e obtiveram aumento do parasitismo de 25 a $30 \%$ para $50 \%$ em estruturas no solo e mais de $80 \%$ em estruturas na planta.

Os predadores e fases de desenvolvimento desses, os quais foram avaliados no estudo são aqueles que apresentam maior associação com o dossel das plantas devido à baixa capacidade de dispersão e, portanto, podendo ser considerados como residentes e alvo do efeito abrasivo do caulim. Assim, por estarem constantemente presentes no dossel das plantas a ação do caulim sobre a sua serosidade, obstrução de espiráculos e desidratação poderia afetá-los, como potenciais efeitos do caulim sobre os artrópodes referidos por Glenn et al. (1999). Maior abundância de Formicidae no tratamento sem caulim, na avaliação em que não foi observado nenhum Formicidae nas plantas com o caulim e maior abundância de Chrysopidae, no tratamento com o caulim na primeira avaliação (Figura 2). De certa forma um resultado favorável à pulverização com o caulim, no caso de Chrysopidae, porém imaturos de Chrysopidae sofrem grande pressão de predação de outros predadores, em especial de formigas (EUBANKS et al., 2002). Assim, esta variação de resultado pode ser uma consequência da abundância de Formicidae no tratamento sem caulim (Figura 2), pois caracteriza um resultado oposto com relação à abundância de Formicidae.

A predação intraguilda é um fenômeno bastante comum em algodoeiro devido à diversidade de predadores e explica a correlação negativa entre a abundância de Chrysopidae com a abundância de joaninhas e aranhas (EUBANKS et al., 2002). Estes grupos de inimigos naturais foram relativamente comuns em ambos os tratamentos em 2011, e menos abundantes em 2010 (Figura 2). Entre as formigas, podemos destacar Solenopsis invicta Buren (Hymenoptera: Formicidae), que é uma espécie frequentemente encontrada associada às colônias de pulgões em algodoeiro (TORRES; RUBERSON, 2006). Esta é citada como predadora de insetos desfolhadores do algodoeiro e do bicudo, mas também sendo uma espécie que interfere com a abundância de outros inimigos naturais, importantes no manejo de pragas do algodoeiro (KAPLAN; EUBANKS, 2002).

Os estudos visando o uso do caulim no controle de pragas têm demonstrado resultados variáveis tanto quanto a sua eficácia (GLENN et al., 1999; 
SHOWLER, 2002; PASCUAL et al., 2010; D'AQUINO et al., 2011), bem como sobre inimigos naturais nos agroecossistemas estudados. Em pomares de maçã, enquanto o tratamento com caulim ocasionou supressão do bicudo da flor Anthonomus pomorum Linnaeus (Coleoptera:, Curculionidae), da mosca serrana Hoplocampa testudinea Klug (Hymenoptera: Tenthredinidae) e da traça-damaçã Cydia pomonella Walsingham (Lepidoptera: Tortricidae); as populações do pulgão Dysaphis plantaginea Passerini (Homoptera: Aphididae), das minadoras das folhas Phyllonorycter blancardellaI Fabricius (Lepidoptera: Gracillariidae) e Lyonetia clerkella Linnaeus (Lepidoptera: Lyonetiidae) e a mosca Phytomyza heringiana Hendel (Diptera: Agromyzidae) foram superiores no tratamento com o caulim (SACKETT; BUDDLE; VINCENT, 2007). Além disso, no tratamento como caulim foi observada redução na abundância relativa de certos grupos de predadores generalistas como alguns grupos de aranhas, coccinelídeos e formigas (SACKETT; BUDDLE; VINCENT, 2007). Resultados esses que corroboram com Markó et al., (2008). Estes autores encontraram redução de algumas pragas principais da macieira como os bicudos, mas com aumento de infestações de afídeos e redução de alguns grupos de predadores generalistas como coleópteros, percevejos e de dermápteros. Também, oliveiras pulverizadas com o caulim apresentaram menores infestações de Bactrocera oleae Rossi (Diptera: Tephritidae), Saissetia oleae Olivier (Hemiptera: Coccidae) e Euphyllura olivina Costa (Hemiptera: Psyllidae), sendo que para cochonilhas a redução de ataque variou entre 45 e $50 \%$ (PASCUAL et al., 2010). Assim, fica evidenciado que o impacto do caulim na redução populacional de pragas e inimigos naturais pode ser variável dependendo da espécie e do agroecossistema. Portanto, deve-se considerar a relevância na redução de pragas chave em cada agroecossistema. Além disso, é notório em vários desses estudos que o caulim possui maior impacto sobre pragas de frutos, como é o caso do bicudo em algodoeiro, além de apresentar outros benefícios, como proteção contra queima dos frutos pelo sol e tolerância ao estresse hídrico e térmico em diferentes culturas (JIFON; SYVERTSEN, 2003; MOFTAH; AL-HUMAID, 2005, SOTELOCUITIVA et al., 2011).

Com base nos resultados encontrados, pode-se concluir que o caulim pulverizado no algodoeiro não causa interferência nos inimigos naturais para o manejo de pragas desta cultura, em especial, no parasitismo do bicudo, que é uma praga limitante no cultivo do algodoeiro e alvo da utilização do caulim. Desta forma, oferecendo uma opção racional e de baixo custo para o cultivo do algodoeiro no sistema de agricultura familiar e orgânico, preconizado para o Semiárido Nordestino (NEVES; TORRES; VIVAN, 2009 NEVES; TORRES, SILVA, 2010; NEVES et al., 2013).

\section{Agradecimentos}

Ao CNPq, a CAPES, e a FACEPE pela concessão de bolsas aos autores, ao Maurício Silva de Lima e ao Adelmo Adriane Santana pelo auxílio nas coletas em campo.

\section{Referências}

ADAMS, C. H.; CROSS, W. H.; MITCHELL, H. C. Biology of Bracon mellitor a parasite of the boll weevil. Journal of Economic Entomology, Lanham, v. 62, n. 4, p. 889-896, 1969.

BASTOS, C. S.; TORRES; J. B. Controle biológico e o manejo integrado de pragas do algodoeiro. Campina Grande: Embrapa Algodão, 2006. 63 p.

BURKS, B. D. Parasitic wasps of the Catolaccus grandis in the Americas. USDA Technology Bulletin, v. 1093, n. 1, p. 1-21, 1954.

D'AQUINO, S.; COCCO, A.; ORTU, S.; SCHIRRA, M. Effects of kaolin-based particle film to control Ceratitis capitata (Diptera: Tephritidae) infestations and postharvest decay in citrus and stone fruit. Crop Protection, v. 30, n. 8, p. 1079-1086, 2011. 
EUBANKS, M. D.; BLACKWELL, S. A.; PARRISH, C. J.; DELAMAR, Z. D.; SANDERS, H. H. Intraguild predation of beneficial arthropods by red imported fire ants in cotton. Environmental Entomology, Lanham, v. 31, n. 6, p. 1168-1174, 2002.

GARCIA, M. E.; BERKETT, L. P.; BRADSHAW, T. Does Surround $\mathbb{R}$ have non-target impacts on New England orchards? In: BRAMLAGE, W. J. (Org.). New England fruit meetings 2002-2003. North Amherst: New England University Cooperative Extensions, 2003. p. 3539.

GLENN, D. M.; PUTERKA, G. J.; VANDERZWET, T.; BYERS, R. E.; FELDHAKE, E. C. Hydrophobic particle films: A new paradigm for suppression of arthropod pests and plant diseases. Journal of Economic Entomology, Lanham, v. 92, n. 4, p. 759-771, 1999.

GLENN, D. M.; PUTERKA, G. Particle films: a new technology for agriculture: horticultural reviews. v. 31. Disponível em: <http://media.wiley.com/product_data/ excerpt/47/04716669/0471666947.pdf>. Acesso em: 19 jun. 2011.

GONZÁLEZ-NÚÑEZ, M.; PASCUAL, S.; SERIS, E.; ESTEBAN-DURÁN, J. R.; MEDINA, P.; BUDIA, F.; ADÁN, A.; VIÑUELA, E. Effects of different control measures against the olive fruit fly (Bactrocera oleae (Gmelin) on beneficial arthropod fauna. Methodology and first results of field assay. IOBC/WPRS Bull., Ghent, v. 35, n. 1, p. 26-31, 2008.

JIFON, J. L.; SYVERTSEN, J. P. Kaolin particle film applications can increase photosynthesis and water use efficiency of 'ruby red' grapefruit leaves. Journal of Amercia Society of Horticultural Science, Alexandria, v. 128, n. 1, p. 104-112, 2003.

KAPLAN, I.; EUBANKS, M. D. Disruption of cotton aphid (Homoptera: Aphididae) - natural enemy dynamics by red imported fire ants (Hymenoptera: Formicidae). Environmental Entomology, Lanham, v. 31, n. 6, p. 11751183, 2002.

MARKÓ, V.; BLOMMERS, L. H. M.; BOGYA, S.; HELSEN, H. Kaolin particle films suppress many apple pests, disrupt natural enemies and promote woolly apple aphid. Journal of Applied Entomology, Lanham, v. 132, n. 1, p. 26-35, 2008.

MOFTAH, A. E.; AL-HUMAID A. I. Effects of kaolin and pinolene film-forming polymers on water relations and photosynthetic rate of tuberose (Polianthes tuberosa L.). Journal of King Saud University Agricultural Science, Riyadh v. 18, n. 1, p. 35-49, 2005.
MORALES-RAMOS, J. A.; ROJAS, M. G.; COLEMAN, R. J.; KING, E. G. Potential use of in vitro Catolaccus grandis (Hymenoptera: Pteromalidae) for biological control of the boll weevil (Coleoptera: Curculionidae). Journal of Economic Entomology, Lanham, v. 91, n. 1, p. 101-109, 1998.

NEVES, R. C. S.; TORRES, J. B.; VIVAN, L. M. Reproduction and dispersal of wing-clipped predatory stinkbugs, Podisus nigrispinus in cotton fields. BioControl, Dordrecht, v. 54, n. 1, p. 9-17, 2009.

NEVES, R. C. S.; TORRES, J. B.; SILVA, M. N. B. Época apropriada para a poda apical visando o controle de pragas do algodoeiro. Pesquisa Agropecuária Brasileira, Brasília, v. 45, n. 12, p. 1342-1350, 2010.

NEVES, R. C. S.; SHOWLER, A. T.; PINTO, E. S.; BASTOS, C. S.; TORRES, J.B. Reducing boll weevil populations by clipping terminal buds and removing abscised fruiting bodies. Entomologia Experimentalis et Applicata, v. 146, n. 2, p. 276-285, 2013.

PASCUAL, S.; COBOS, G.; SERIS, E.; GONZÁLEZNÚÑEZ, M. Effects of processed kaolin on pests and non-target arthropods in a Spanish olive grove. Journal of Pesticide Science, London, v. 83, n. 2, p. 121-133, 2010.

RAMALHO, F. S.; JESUS, F. M. M.; BLEICHER, E. Manejo integrado de pragas e viabilidade do algodoeiro herbáceo no Nordeste. In: SEMINÁRIO SOBRE CONTROLE DE INSETOS, 1., 1989. Campinas. Anais... Campinas: Fundação Cargil, 1989. p. 112-123.

RAMALHO, F. S.; MEDEIROS, R. S.; LEMOS, W. P.; WANDERLEY, P. A.; DIAS, J. M.; ZANUNCIO, J. C. Evaluation of Catolaccus grandis (Burks) (Hym.: Pteromalidae) as a biological control agent against cotton boll weevil. Journal of Applied Entomology, Lanham, v. 124, n. 1, p. 9-10, 2000.

RAMALHO, F. S.; SILVA, J. R. B. Período de emergência e mortalidade natural do bicudo-do-algodoeiro. Pesquisa Agropecucária Brasileir, Brasília, v. 28, n. 11, p. 12211231, 1993.

RAMALHO, F. S.; WANDERLEY, P. A. Ecology and management of the boll weevil in South American cotton. American Entomologist, Lanham, v. 42, n. 1, p. 41-47, 1996.

SACKETT, T. E.; BUDDLE, M.; VINCENT, E. C. Effects of kaolin on the composition of generalist predator assemblages and parasitism of Choristoneura rosaceana (Lep., Tortricidae) in apple orchards. Journal of Applied Entomology, Berlin, v. 131, n. 7, p. 478-485, 2007.

SAS INSTITUTE. SAS/STAT User's guide, version 8.02, TS level 2MO. Cary: SAS Institute Inc., 2001. 
SHOWLER, A. T. Effects of kaolin-based particle film application on boll weevil (Coleoptera: Curculionidae) injury to cotton. Journal of Economic Entomology, Lanham, v. 95, n. 4, p. 754-762, 2002.

Relationships of different cotton square sizes to boll weevil (coleoptera: Curculionidae) feeding and oviposition in field conditions. Journal of Economic Entomology, Lanham, v. 98, n. 5, p. 1572-1579, 2007.

SHOWLER, A. T.; SÉTAMOU, M. Effects of kaolin particle film on selected arthropod populations in cotton in the lower Rio Grande Valley of Texas. Southwestern Entomologist, Canyon, v. 29, n. 2, p. 137-146, 2004.

SILVA, C. A. D.; RAMALHO, F. S. Kaolin spraying protects cotton plants against damages by boll weevil Anthonomus grandis Boheman (Coleoptera: Curculionidae). Journal of Pest Science, Dordrecht, v. 88 , n. 3, p. 563-569, 2013.

SOTELO-CUITIVA, Y. M.; RESTREPO-DÍAZ, H.; GARCÍA-CASTRO, A.; RAMÍREZ-GODOY, A.; FLÓREZ-RONCANCIO, V. J. Effect of kaolin film particle applications (Surround $\mathrm{WP}^{\circledR}$ ) and water deficit on physiological characteristics in rose cut plants (Rose spp.). American Journal of Plant Science, Irvine, v. 2, n. 3, p. 354-358, 2011.
TORRES, J. B.; RUBERSON, J. R. Abundance and diversity of ground-dwelling arthropods of pest management importance in commercial $\mathrm{Bt}$ and non-Bt cotton fields. Annals of Applied Biology, Warwick, v. 150, n. 1, p. 27-39, 2006.

TORRES, J. B.; RUBERSON, J. R.; WHITEHOUSE, $M$. Transgenic cotton for sustainable pest management. In: LICHTFOUSE, E. (Org.). Sustainable agriculture reviews: organic farming, pest control and remediation of soil pollutants. New York: Springer, 2009. p. 45-82.

WANDERLEY, P. A. Biologia e exigências térmicas de Bracon vulgaris Ashmead (Hymenoptera: Braconidae), parasitoide do bicudo-do-algodoeiro (Anthonomus grandis Boheman) (Coleoptera: Curculionidae). 1998. Dissertação (Mestrado em Fitossanidade) - Universidade Federal Rural de Pernambuco, Recife.

WHITCOMB, W. H.; BELL, K. Predaceous insects, spiders, and mites of Arkansas cotton fields. Fayetteville: Agricultural Experiment Station University of Arkansas Bulletin, 1964. $690 \mathrm{p}$. 